\title{
Análise da Fadiga Relatada e das Forças Musculares Respiratória e Periférica em indivíduos com Câncer em Tratamento
}

\author{
doi: https://doi.org/10.32635/2176-9745.RBC.2020v66n4.1051
}

\author{
Analysis of Reported Fatigue and of Respiratory and Peripheral Muscle Strength in Individuals with Cancer Undergoing Treatment \\ Análisis de la Fatiga Reportada e de las Fuerzas Musculares Respiratoria y Periférica en Individuos con Cáncer Sometidos \\ a Tratamiento
}

Karina Oliveira Prado Mariano'; Ricardo da Silva Alves²; Ana Paula Aparecida Mantuani ${ }^{3}$; Sabrina Rosse Carvalho ${ }^{4}$ Carmélia Bomfim Jacó Rocha ${ }^{5}$; Denise Hollanda Iunes ${ }^{6}$; Juliana Bassalobre Carvalho Borges ${ }^{7}$; Leonardo Cesar Carvalho ${ }^{8}$

\begin{abstract}
RESUMO
Introduçáo: Nos últimos anos, o tratamento do câncer evoluiu, possibilitando uma maior sobrevida ao paciente, porém os efeitos colaterais, como a diminuiçáo da imunidade e a fadiga, influenciam o sistema respiratório e muscular. Objetivo: Analisar a fadiga e as forças musculares respiratória e periférica em voluntários com câncer em tratamento e em indivíduos saudáveis. Método: Trata-se de um estudo analítico, observacional, transversal e controlado. Os indivíduos foram distribuídos em dois grupos: um grupo de câncer em quimioterapia e/ou em radioterapia (GCA: $\mathrm{n}=98$; homens $=35,72 \%$; mulheres $=64,28 \%$; idade $=58,13 \pm 13,26$ anos; índice de massa corporal $\left(\mathrm{IMC}=26,23 \pm 4,04 \mathrm{~kg} / \mathrm{m}^{2}\right.$; tempo de diagnóstico de câncer $=27,54 \pm 9,61$ meses) e um grupo controle (GC: $n=86$; homens=30,23\%; mulheres=69,77; idade=59,24 $\pm 12,87$ anos; IMC $=26,76 \pm 4,04 \mathrm{~kg} / \mathrm{m}^{2}$ ). Para todos os indivíduos, a fadiga relatada foi avaliada, usando-se a subescala de fadiga do questionário The Functional Assessment of Cancer Therapy-Fatigue (FACT-F). A avaliação das pressōes respiratórias máximas foi realizada por meio da manovacuometria e da força de preensão palmar por intermédio da dinamometria manual. Resultados: O GCA apresentou maior índice de fadiga relatada $\left(\mathrm{p}<0,001 ; \mathrm{f}^{2}=0,382\right)$, valores inferiores para as variáveis respiratórias (PImax: $\mathrm{p}<0,001 ; \mathrm{f}^{2}=0,441 ; \mathrm{PEmax}: \mathrm{p}<0,001 ; \mathrm{f}^{2}=0,361$ ), força de preensão palmar esquerda $\left(\mathrm{p}=0,024 \mathrm{f}^{2}=0,182\right)$, se comparado ao GC. Conclusão: Voluntários com câncer em quimioterapia e/ou em radioterapia apresentaram maiores níveis de fadiga relatada, com reduçôes da força muscular respiratória e da força de preensão palmar. Palavras-chave: Neoplasias; Força Muscular; Fadiga Muscular; Teste respiratórios; Reabilitaçấo.
\end{abstract}

\section{ABSTRACT}

Introduction: In recent years, cancer treatment has evolved, allowing longer survival for the patient, but, side effects such as a decreased immunity and fatigue influence the respiratory and muscular systems. Objective: Analyze fatigue, the respiratory and peripheral muscle strength in individuals with cancer undergoing treatment and in healthy individuals. Method: Analytical, observational, cross-sectional, and controlled study. The individuals were divided into two groups: a cancer group in chemotherapy and/or radiotherapy $($ GCA: $\mathrm{n}=98 ;$ men $=35.72 \%$; women $=64.28 \%$; age $=58.13$ \pm 13.26 years; body mass index $(B M I)=26.23 \pm 4.04 \mathrm{~kg} / \mathrm{m}^{2}$; time of cancer diagnosis $=27.54 \pm 9.61$ months $)$ and a control group (CG: $\mathrm{n}=86$; men $=$ $30.23 \%$; women $=69.77$; age $=59.24 \pm 12.87$ years; $\mathrm{BMI}=26.76 \pm 4.04$ $\mathrm{kg} / \mathrm{m}^{2}$ ). For all the individuals, the reported fatigue was assessed using the fatigue subscale of The Functional Assessment of Cancer Therapy-Fatigue (FACT-F) questionnaire. The evaluation of maximal respiratory pressures was performed by manovacuometry and the palmar grip strength by manual dynamometry. Results: The GCA showed the highest reported fatigue index $\left(\mathrm{p}<0.001 ; \mathrm{f}^{2}=0.382\right)$, lower values for respiratory variables (PImax: $\mathrm{p}<0.001$; $\mathrm{f}^{2}=0.441$; PEmax: $\left.\mathrm{p}<0.001 ; \mathrm{f}^{2}=0.361\right)$, left palmar grip strength $(\mathrm{p}=0.024$; $\mathrm{f}^{2}=0.182$ ), when compared to the CG. Conclusion: Volunteers with cancer in chemotherapy and/or radiotherapy presented higher levels of reported fatigue, with reductions in respiratory muscle strength and palmar grip strength. Key words: Neoplasms; Muscle Strength; Muscle Fatigue; Breath Tests; Rehabilitation.

\section{RESUMEN}

Introducción: En los últimos años, el tratamiento oncológico ha evolucionado, permitiendo una supervivencia más larga para el paciente, pero los efectos secundarios como la disminución de la inmunidad y la fatiga influyen en los sistemas respiratorios y musculares. Objetivo: Analizar la fatiga e las fuerzas musculares respiratoria y periférica en individuos con cáncer sometidos a tratamiento y en individuos sanos. Método: Se trata de un estudio analítico, observacional, transversal y controlado. Los individuos fueran divididos en dos grupos: un grupo de cáncer bajo quimioterapia y/o radioterapia (GCA: no 98; hombres $35,72 \%$; mujeres $64,28 \%$; edad $58,13 \pm 13,26$ ańos; indice de masa

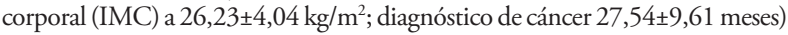
y un grupo de control (CG: no 86; hombres 30,23\%; mujeres 69,77; edad de 59,24 12,87 ańos; IMC $26,76 \pm 4,04 \mathrm{~kg} / \mathrm{m}^{2}$ ). Para todos los voluntarios, la fatiga notificada se evaluó utilizando la subescala de fatiga del cuestionario The Functional Assessment of Cancer Therapy-Fatigue (FACT-F). La evaluación de las presiones respiratorias máximas se realizó mediante manovacuometría y fuerza de agarre palmar mediante dinamometría manual. Resultados: El GCA tuvo el índice de fatiga notificado más alto $\left(\mathrm{p}<0,001 ; \mathrm{f}^{2}=0,382\right)$, valores más bajos para las variables respiratorias (PImax: $p<0,001 ; f^{2}=0,441$; PEmax: $\left.\mathrm{p}<0,001 ; \mathrm{f}^{2}=0,361\right)$, fuerza de agarre palmar izquierda $(\mathrm{p}=0,024$; $\left.\mathrm{f}^{2}=0,182\right)$, en comparación con el GC. Conclusión: Voluntarios con cáncer en quimioterapia y/o radioterapia presentaron mayores niveles de fatiga reportados, con reducciones en la fuerza muscular respiratoria y fuerza de agarre palmar. Palabras clave: Neoplasias; Fuerza Muscular; Fatiga Muscular; Pruebas Respiratorias; Rehabilitación.

'Departamento de Fisioterapia da Fundação de Ensino e Tecnologia de Alfenas da Universidade José do Rosário Vellano (Unifenas). Alfenas (MG), Brasil. Orcid iD: https://orcid.org/0000-0003-3007-2833

${ }^{2}$ Faculdade de Fisioterapia da Universidade do Vale do Sapucaí (Univás). Programa de Pós-Graduação em Ciências da Reabilitação e Biociências Aplicadas à Saúde. Alfenas (MG), Brasil. Orcid iD: https://orcid.org/0000-0001-6931-3943

${ }^{3}$ Instituto de Ciências da Motricidade da Universidade Federal de Alfenas (Unifal). Programa de Pós-Graduação em Ciências da Reabilitação. Alfenas (MG), Brasil. Orcid iD: https://orcid.org/0000-0002-5989-8981

${ }_{4}^{4}$ Instituto de Ciências da Motricidade da Unifal. Alfenas (MG), Brasil. Orcid iD: https://orcid.org/0000-0002-8632-3585

IInstituto de Ciências da Motricidade da Unifal. Alfenas (MG), Brasil. Orcid iD: https://orcid.org/0000-0002-7819-5683

'Instituto de Ciências da Motricidade da Unifal. Programa de Pós-Graduação em Ciências da Reabilitação. Alfenas (MG), Brasil. Orcid iD: https://orcid.org/0000-0003-1396-9980 'Instituto de Ciências da Motricidade da Unifal. Programa de Pós-Graduação em Ciências da Reabilitação. Alfenas (MG), Brasil. Orcid iD: https://orcid.org/0000-0003-0868-717X ${ }^{8}$ Instituto de Ciências da Motricidade da Unifal. Programa de Pós-Graduação em Ciências da Reabilitação e Biociências Aplicadas à Saúde. Alfenas (MG), Brasil. Orcid iD: https://orcid.org/0000-0001-6511-8315

Endereço para correspondência: Karina Oliveira Prado Mariano. Rua Salvador Quesada, 65 - Jardim Aeroporto. Alfenas (MG), Brasil. CEP 37130-804. E-mail: drakarina@bol.com.br 


\section{INTRODUÇÃO}

O câncer é considerado uma doença crônica, uma das principais causas de morte e obstáculo para o aumento da expectativa de vida em todos os países do mundo no século XXI ${ }^{1}$. No Brasil, houve aumento da incidência e da mortalidade por câncer, enquanto, em países desenvolvidos, mesmo apresentando aumento da incidência, houve redução da mortalidade ${ }^{2}$.

O tratamento para os diversos tipos de câncer, geralmente, é cirúrgico e acompanhado de quimioterapia e/ou de radioterapia e/ou de hormonioterapia el ou imunoterapia ${ }^{3}$. Os efeitos dessas modalidades de tratamento induzem ao aumento da atividade de citocinas que levam a alteraçóes como a sarcopenia, caquexia, aumento da fadiga muscular ${ }^{4,5}$. A ativação desses fatores leva a alteraçóes no metabolismo proteico muscular, acarretando aumento da degradação e diminuição da síntese, o que pode predispor à redução na capacidade de gerar força muscular ${ }^{6}$.

A avaliação da força muscular periférica pode ser realizada por meio do teste de preensão palmar e tem sido utilizada como um determinante de fragilidade, relacionando-a com a fadiga muscular global ${ }^{7,8}$ e como um marcador de prognóstico de mortalidade em diversas faixas etárias ${ }^{9}$. Alguns estudos sugerem que a presença da fadiga pode refletir em um comprometimento das forças muscular periférica e muscular respiratória ${ }^{10-12}$.

Um dos principais efeitos ocasionados pelo tratamento quimioterapêutico e radioterapêutico é a fadiga relacionada ao câncer (FRC), que afeta $95 \%$ dos pacientes ${ }^{13}$, podendo permanecer por meses a anos após o seu términot. A FRC, de acordo com o National Comprehensive Cancer Network, é definida como um sintoma "angustiante, persistente e subjetiva sensação de cansaço físico, emocional e/ ou cognitivo ou exaustáo relacionada ao câncer ou tratamento de câncer que não é proporcional à atividade recente realizada e interfere no funcionamento normal"' . Alguns estudos têm demonstrado que pacientes com câncer em estágios diferentes do tratamento apresentam, além da FRC $^{14,15}$, reduçáo da força muscular periférica ${ }^{16}$, como dos músculos da perna ${ }^{14}$ e do músculo deltoide médio ${ }^{15}$.

Os tratamentos para os diferentes tipos de câncer podem provocar danos ao tecido epitelial, diminuiçáo da capacidade inspiratória e da capacidade vital, interferindo no volume pulmonar e na força muscular respiratória ${ }^{17,18}$. A presença de FRC também pode ter impacto negativo no funcionamento do sistema respiratório, acarretando disfunçôes ventilatórias ${ }^{19}$. Além disso, a quimioterapia pode contribuir para a aparição de sintomas neurológicos ${ }^{20}$. Já a radioterapia favorece a restrição da mobilidade, a diminuição da capacidade de contraçáo dos músculos respiratórios com consequente alteração nas pressóes respiratórias ${ }^{17,21}$ e no volume pulmonar ${ }^{17}$.
Um método útil para a avaliação das pressôes respiratórias máximas é a manovacuometria, visto que a pressão inspiratória máxima (PImax) apresenta importante relevância clínica, pois os músculos inspiratórios suportam maiores cargas de trabalho ventilatório. As mensuraçōes da PImax e da pressão expiratória máxima (PEmax) são úteis para diferenciar a fraqueza neuromuscular de músculos abdominais e uma fraqueza específica do diafragma ou de outros músculos respiratórios ${ }^{22}$.

Outro fator relacionado com a acentuação da percepção da fadiga pode ser atribuído às orientaçóes dos profissionais de saúde que incentivam os pacientes a permanecerem em repouso durante e após o tratamento ${ }^{23,24}$. Nesse sentido, o presente estudo busca ofertar uma melhor compreensão sobre a fadiga relatada, a força muscular periférica e a força muscular respiratória em voluntários com câncer em tratamento, comparados a voluntários sem câncer.

\section{MÉTODO}

Trata-se de um estudo analítico, observacional, transversal e controlado, aprovado pelo Comitê de Ética em Pesquisa da Universidade Federal de Alfenas (Unifal), MG, número 1.980.365, realizado de agosto de 2016 a abril de 2018. Todos os voluntários foram esclarecidos a respeito dos objetivos do estudo e assinaram o Termo de Consentimento Livre e Esclarecido.

A amostra foi alocada por conveniência, sendo dividida em dois grupos: o de voluntários com câncer em tratamento (GCA) e o de voluntários sem câncer - grupo controle (GC). O GC foi constituído por acompanhantes de pacientes que estavam na clínica de fisioterapia, recrutados aleatoriamente por meio de convite para participar deste estudo e pareados ao GCA pela idade, massa corporal, altura e sexo.

Os critérios de inclusão para o GCA foram: voluntários de ambos os sexos, acima de 45 anos, com diagnóstico clínico de câncer em diferentes estádios ( 0 a IV) e que se encontravam em tratamento com quimioterapia el ou radioterapia. Para o GC, foram incluídos voluntários de ambos os sexos, acima de 45 anos, que não estavam em uso de antidepressivos e os que não apresentavam doenças crônicas como fibromialgia, hipotireoidismo, insuficiência cardíaca, insuficiência renal crônica, diabetes mellitus, doenças pulmonares, e que nunca tivessem sido diagnosticados com algum tipo de câncer.

Foram excluídos do GCA voluntários de ambos os sexos que apresentaram distúrbios cognitivos que restringiam a explicação sobre o manuseio dos sistemas de avaliação. Além disso, também foram excluídas pessoas internadas, traqueostomizadas, aquelas com diagnóstico médico de câncer de pulmão ou com metástase no pulmão, e as que não conseguiram realizar a avaliação. Foram 
excluídos do GC os voluntários que não conseguiram realizar a avaliação.

Os voluntários do GCA foram avaliados na sala de espera da clínica de oncologia da Santa Casa de Alfenas, MG, onde aguardavam consulta médica com o oncologista, e os voluntários do GC foram avaliados na clínica de fisioterapia da Unifal, MG.

Realizou-se um estudo-piloto para o cálculo do tamanho da amostra ( $G^{*}$ Power software, v. 3.1.7, Franz Faul, Universität Kiel, Germany). O N amostral estimado para um poder de $80 \%$ foi de 80 voluntários por grupo para a variável Functional Assessment of Cancer Therapy-Fatigue (FACT-F). No total, foram avaliados 98 voluntários no GCA e 86 no GC.

A altura e a massa corpórea foram aferidas por uma balança mecânica antropométrica equipada com estadiômetro, ambos da marca Welmy ${ }^{\circledR}$. O índice de massa corpórea (IMC) foi obtido a partir dos valores de massa corporal $(\mathrm{Kg})$, dividido pela estatura ao quadrado $\left(\mathrm{m}^{2}\right)$, classificados segundo a Organização Mundial da Saúde (OMS) 25 .

Para determinar as variáveis de frequência cardíaca e respiratória, os voluntários permaneceram sentados em repouso. Foram observados os movimentos do tórax ou da parede abdominal e contado o número de incursóes respiratórias em um minuto, determinado a frequência respiratória. A frequência cardíaca foi mensurada pela aferiçáo do pulso na artéria radial por palpação durante um minuto ${ }^{26}$.

$\mathrm{Na}$ avaliação da força muscular respiratória, utilizou-se o manovacuômetro da marca Comercial Médica (Indústria Brasileira, intervalo de medida $0 \pm 120 \mathrm{~mm} \mathrm{H}_{2} \mathrm{O}$ ). Foram verificadas as variáveis de PImax e PEmax.

O voluntário, após receber instruções prévias sobre a técnica, foi orientado a permanecer na posição sentada, com os clipes nasal e bucal entre os dentes, com os lábios bem fechados em torno destes, para não permitir escape de ar. Para a obtenção da PImax, realizou-se a manobra de inspiração forçada a partir do volume residual. E, posteriormente, uma expiração forçada a partir da capacidade pulmonar total para a obtençáo da PEmax. Os procedimentos foram repetidos por três vezes consecutivas para a PImax, seguida da PEmax, permitindo um minuto de repouso entre os esforços e registrado o maior valor; as pressóes foram sustentadas aproximadamente por um segundo, sob comando verbal ${ }^{27}$.

Para a análise da fraqueza muscular respiratória, consideraram-se apenas os valores da PImax, sendo $\leq-70 \mathrm{~cm} \mathrm{H}_{2} \mathrm{O}$ força aceitável e $\geq-69 \mathrm{~cm} \mathrm{H}_{2} \mathrm{O}$ como não aceitável ${ }^{28}$, estratificando, dessa forma, a amostra em dois subgrupos.

A avaliação da força muscular periférica foi realizada com um dinamômetro de mão da marca JAMAR ${ }^{\circledR}$ (Patterson
Medical co. EUA), com o indivíduo confortavelmente sentado, encostado e sem apoio para os membros superiores. Foi medida a força de preensão palmar direita (FPPD) e esquerda (FPPE). A posiçáo do membro superior para a execução do exame foi mantida com o ombro aduzido e rotação neutra, cotovelo flexionado a $90^{\circ}$, antebraço na posição neutra, punho entre $0^{\circ}$ e $30^{\circ}$ de flexão e com $0^{\circ}$ a $15^{\circ}$ de desvio ulnar. Solicitaram-se três contraçôes voluntárias máximas para cada membro, com repouso de um minuto entre as repetiçóes e considerada a maior medida obtida para cada membro ${ }^{29}$.

Para a avaliação da fadiga e suas repercussôes, utilizou-se o questionário FACT-F, instrumento validado e traduzido para a língua portuguesa, sendo aplicável em pacientes com câncer e na população em geral sem câncer ${ }^{30}$.

Com esse questionário, é possível realizar o cálculo da subescala de fadiga que contempla 13 itens específicos para avaliar a fadiga com escore de 0 a 52; quanto maior a pontuaçáo, menor a fadiga dos avaliados ${ }^{30}$. $\mathrm{Na}$ determinação para a presença de fadiga, utilizou-se o ponto de corte de 43 pontos na subescala de fadiga do FACT-F. Esse valor possui sensibilidade de 0,92 e especificidade de 0,69 , quando comparados os pacientes com câncer e uma populaçấo saudável ${ }^{31}$. Dessa forma, considerou-se fadiga quando houve a ocorrência de valores inferiores ao ponto de corte.

Para a análise estatística dos dados, utilizou-se o software Statistical Package for the Social Sciences (SPSS) (IBM Corp., Chicago, EUA), versão 20.0.

Inicialmente, os dados foram analisados por meio de métodos estatísticos descritivos, sendo obtidos os valores de média, desvio-padrão (DP) e intervalo de confiança (IC 95\%). Em seguida, todos os conjuntos de dados da amostra foram testados quanto à sua normalidade por meio do teste Kolmogorov-Smirnov. Após essa análise, realizou-se o teste de análise de variância (Anova) one way quando a amostra foi normal. Caso esta não apresentasse normalidade, seria submetida ao teste Kruskal-Wallis. Para os dados categóricos nominais, utilizou-se o teste qui-quadrado.

Em relação às variáveis independentes, ao atender ao critério de distribuição normal, procedeu-se à condução de teste $t$ de Student e, quando não atendido esse critério, utilizou-se o teste $U$ de Mann-Whitney, para a comparação intergrupos. Para ambos os testes, foi considerado um nível de significância de 5\%.

Além disso, para todas as variáveis, calculou-se o poder da amostra, sendo este considerado a partir de 0,80 um alto poder $^{32}$. Para calcular o tamanho de efeito do Anova, utilizou-se $\mathrm{f}$ de Cohen, sendo considerados os valores de 0,02 a 0,15 (pequeno efeito), 0,15 a 0,35 (efeito mediano) 
e acima de 0,35 (efeito grande) ${ }^{32}$. Para calcular o tamanho de efeito do teste $t$, o d de Cohen foi utilizado e adotados os valores entre 0,01 a 0,2 (pequeno efeito), 0,21 a 0,79 (efeito mediano) e igual ou acima de 0,80 (efeito grande) ${ }^{33}$. Para todas as variáveis analisadas neste estudo, adotou-se um nível de significância de $\mathrm{p}<0,05$.

\section{RESULTADOS}

Inicialmente, avaliaram-se 102 voluntários no grupo câncer, com exclusão de quatro indivíduos com diagnóstico de câncer de pulmão, totalizando a amostra final com 98 voluntários. Destes, 69 realizaram quimioterapia e 29 realizaram quimioterapia e radioterapia. Para o grupo controle, avaliaram-se 94 voluntários; dos quais, oito foram excluídos, dois por fibromialgia, três por hipotireoidismo e três por diabetes, totalizando a amostra 86 indivíduos.

As características sociodemográficas e clínicas dos voluntários encontram-se na Tabela 1 e demonstram homogeneidade entre os grupos.

A Tabela 2 apresenta a análise comparativa entre GCA e GC para as variáveis de frequência cardíaca, frequência respiratória, forças musculares respiratória e periférica. É possível observar que os voluntários do GCA apresentam maior frequência cardíaca, redução dos valores de PEmax, PImax e FPPE, se comparados aos do GC. Ao se considerar a subescala de fadiga, observou-se que os voluntários do GCA se apresentavam significativamente fadigados em comparação aos do GC (ponto de corte da subescala de fadiga $=43$ pontos) .

As análises das variáveis dentro do GCA e do GC, segundo os subgrupos de força muscular respiratória aceitável e não aceitável, estão apresentadas nas Tabelas 3 e 4.

Ao analisar as variáveis respiratórias e de FPP dos voluntários do GCA pela classificação da força muscular respiratória aceitável e não aceitável, observam-se valores mais baixos nos voluntários de força não aceitável em relaçáo às variáveis PEmax, PImax, FPPD, FPPE do que nos voluntários de força aceitável. O número de voluntários do GCA que apresentam força não aceitável é $21,81 \%$ maior do que os que apresentam força aceitável. Não existe diferença entre os voluntários do GCA que possuem força muscular respiratória aceitável e não aceitável quanto à subescala de fadiga (Tabela 3).

Ao analisar as variáveis respiratórias e de FPP dos voluntários do GC que apresentam força muscular respiratória aceitável ou não aceitável, observam-se valores mais baixos nos voluntários de força não aceitável em relação às variáveis PEmax, PImax, FPPD, FPPE do que nos voluntários de força aceitável. O número de voluntários do GC que apresentam força não aceitável é $73,52 \%$ menor do que os que apresentam força aceitável. Existe diferença entre os voluntários do GC que possuem força muscular respiratória aceitável e não aceitável quanto à subescala de fadiga (Tabela 4).

\section{DISCUSSÃO}

O tratamento antineoplásico é fundamental para os diversos tipos de câncer, o que resulta na melhora da qualidade de vida. Todavia, oferece potenciais riscos de toxicidade, por causar efeitos colaterais, como alteraçóes cardiovasculares ${ }^{23,34,35}$.

Um aumento significativo da frequência cardíaca no GCA, em comparação ao GC, foi observado. Acredita-se que essa alteração pode ter sido induzida pela quimioterapia, embora os tipos de quimioterápicos e suas açôes não tenham sido objetos de investigação no presente estudo. Esses fármacos são capazes de enfraquecer o músculo cardíaco (cardiomiopatia) e de induzir distúrbios como arritmias supraventriculares e ventriculares ${ }^{34,35}$, além de danificarem o sistema respiratório ${ }^{10,12,18}$.

A força muscular respiratória pode estar comprometida em pacientes com câncer tratados com quimioterapia ${ }^{17,36}$. Tal fato justifica a investigação das pressōes respiratórias máximas $^{36}$, pois a redução de seus valores demonstra a presença de fraqueza muscular ${ }^{35}$ e poderiam se relacionar com a fadiga relatada pelos investigados ${ }^{37}$. No presente estudo, a quimioterapia e/ou a radioterapia promoveu a redução das forças musculares inspiratória e expiratória. Essa fraqueza pode estar associada às modificaçóes no parênquima pulmonar, além do cansaço e do esgotamento físicos ocasionados pelo tratamento antineoplásico ${ }^{12,17,38}$.

Em outro estudo, ao se comparar pacientes com câncer e pacientes com outras doenças, observou-se redução da função pulmonar e da força muscular periférica ${ }^{18}$. Esses dados corroboram os achados do presente estudo.

A FPP é um indicador geral de força e de potência muscular periférica e se relaciona com taxas de morbidade e de mortalidade ${ }^{8,18}$. Além disso, essa variável se associa às doenças cardiovasculares e ao câncer ${ }^{39}$, e seu declínio demonstra a redução da autonomia para a realização das atividades de vida diária ${ }^{23}$. A quimioterapia e/ou radioterapia reduziu as forças de preensão palmar em ambos os membros avaliados, porém isso não foi significativo para o membro direito. É possível que a concentração de voluntários com diagnóstico de câncer de mama com o lado esquerdo afetado ( $\mathrm{n}=19$, dado náo avaliado) possa ter influenciado os achados. Outra consideração do que pode ter influenciado o resultado foi a de que, em ambos os grupos, esses valores eram inferiores aos de referência populacional brasileira ${ }^{40}$. 
Tabela 1. Características clínicas e sociodemográficas dos voluntários do estudo

\begin{tabular}{|c|c|c|c|}
\hline Varióveis & $\begin{array}{c}\text { GCA }(\mathbf{n}=98) \\
\text { Média (DP) } \\
(\text { IC } 95 \%)\end{array}$ & $\begin{array}{c}\text { GC }(n=86) \\
\text { Média (DP) } \\
\text { (IC 95\%) }\end{array}$ & Valor de $p$ \\
\hline Idade (anos)* & $\begin{array}{l}58,13(13,26) \\
(55,34-60,92)\end{array}$ & $\begin{array}{l}59,24(12,87) \\
(56,41-62,07)\end{array}$ & 0,524 \\
\hline $\operatorname{IMC}\left(\mathrm{kg} / \mathrm{m}^{2}\right)^{*}$ & $\begin{array}{c}26,23(4,04) \\
(25,87-27,65)\end{array}$ & $\begin{array}{c}26,76(4,04) \\
(25,87-27,65)\end{array}$ & 0,336 \\
\hline Massa corporal $(\mathrm{kg})^{*}$ & $\begin{array}{l}67,66(13,15) \\
(64,89-70,44)\end{array}$ & $\begin{array}{c}70,21(11,28) \\
(67,74-72,70)\end{array}$ & 0,162 \\
\hline Altura $(\mathrm{m})^{*}$ & $\begin{array}{l}1,61(0,09) \\
(1,59-1,63)\end{array}$ & $\begin{array}{l}1,62(0,85) \\
(1,60-1,64)\end{array}$ & 0,273 \\
\hline $\begin{array}{ll}\text { Sexo }(\%)^{*} & M \\
& F\end{array}$ & $\begin{array}{l}35,72 \\
64,28\end{array}$ & $\begin{array}{l}30,23 \\
69,77\end{array}$ & 0,376 \\
\hline Tempo de diagnóstico (meses) & $\begin{array}{c}27,54(9,61) \\
(19,59-35,48)\end{array}$ & - & - \\
\hline Diagnóstico de câncer - \%(n) & & - & - \\
\hline Trato gastrointestinal & $26,53 \%(26)$ & - & - \\
\hline Mama & $27,55 \%(27)$ & - & - \\
\hline Abdominopélvico & $28,57 \%(28)$ & - & - \\
\hline Orofaringe & $05,10 \%(05)$ & - & - \\
\hline Outros & $12,25 \%(12)$ & - & - \\
\hline Estádios - \% (n) & & - & - \\
\hline 0 & $26,54 \%(26)$ & - & - \\
\hline I & $8,16 \%(08)$ & - & - \\
\hline II & $29,59(29)$ & - & - \\
\hline III & $25,51(25)$ & - & - \\
\hline IV & $10,20(10)$ & - & - \\
\hline Tipos de tratamento $\%(n)^{\#}$ & & - & \\
\hline Quimioterapia & $(70,40) 69$ & - & \\
\hline Quimioterapia/Radioterapia & $(29,60) 29$ & - & \\
\hline \multicolumn{4}{|l|}{ Presença de fadiga - \%(n)\# } \\
\hline Sim & $67,35(62)$ & 33,72 (29) & 0,009 \\
\hline Não & $32,65(36)$ & $66,28(57)$ & 0,029 \\
\hline \multicolumn{4}{|l|}{ CFMR \% (n) } \\
\hline Aceitável & $42,88(42)$ & $79,06(68)$ & 0,013 \\
\hline Nao aceitável & $57,12(56)$ & $20,94(18)$ & $<0,001$ \\
\hline
\end{tabular}

Legendas: DP: desvio-padrão; IC: intervalo de confiança; IMC: índice de massa corporal; M: masculino; F: feminino; CFMR: classificação de força muscular respiratória. Outros: (leucemia, linfoma, câncer ósseo, câncer encefálico e câncer de pele).

Nota: *teste Anova; \# $\chi^{2}$ : teste qui-quadrado.

Os efeitos da FRC são multidimensionais e podem interferir em todos os aspectos da vida de uma pessoa, incluindo a independência, a força muscular, a concentração e as relaçôes sociais, e podem ser extremamente angustiantes ${ }^{5,13}$.
Os voluntários em tratamento de quimioterapia e/ou em radioterapia alteraram o nível médio de FRC. Tem sido relatado que sobreviventes ao câncer experimentaram um aumento significativo na fadiga após a quimioterapia, radioterapia, hormônio e/ou terapias biológicas ${ }^{41}$. 
Tabela 2. Análise comparativa da frequência cardíaca, frequência respiratória, subescala de fadiga e força muscular respiratória e periférica dos grupos câncer e controle

\begin{tabular}{|c|c|c|c|c|}
\hline Variáveis & $\begin{array}{c}\text { GCA }(n=98) \\
\text { Média (DP) } \\
(\text { IC 95\%) }\end{array}$ & $\begin{array}{c}\text { GC }(n=86) \\
\text { Média (DP) } \\
(\text { IC } 95 \%)\end{array}$ & Valor de $p$ & $\mathbf{f}^{2}$ \\
\hline FC (bpm) & $\begin{array}{l}77,11(12,68) \\
(74,44-79,78)\end{array}$ & $\begin{array}{l}71,17(11,28) \\
(68,69-73,64)\end{array}$ & $0,002^{*}$ & 0,162 \\
\hline FR (irpm) & $\begin{array}{c}17,04(4,27) \\
(16,14-17,94)\end{array}$ & $\begin{array}{c}17,86(4,04) \\
(16,97-18,75)\end{array}$ & 0,086 & 0,098 \\
\hline $\operatorname{PEmax}\left(\mathrm{cm} / \mathrm{H}_{2} \mathrm{O}\right)$ & $\begin{array}{l}75,54(28,86) \\
(69,46-81,62) \\
\end{array}$ & $\begin{array}{c}94,88(24,57) \\
(89,48-100,28)\end{array}$ & $<0,001^{*}$ & 0,361 \\
\hline $\operatorname{PImax}\left(\mathrm{cm} / \mathrm{H}_{2} \mathrm{O}\right)$ & $\begin{array}{l}-67,71(32,60) \\
(60,84-74,57)\end{array}$ & $\begin{array}{c}-93,31(25,25) \\
(87,77-98,86)\end{array}$ & $<0,001^{*}$ & 0,441 \\
\hline FPPD (kgf) & $\begin{array}{l}23,60(10,24) \\
(21,44-25,76)\end{array}$ & $\begin{array}{c}26,13(9,85) \\
(23,97-28,29)\end{array}$ & 0,110 & 0,126 \\
\hline FPPE (kgf) & $\begin{array}{c}21,48(9,51) \\
(19,48-23,48)\end{array}$ & $\begin{array}{c}24,89(9,19) \\
(22,87-26,91)\end{array}$ & $0,024^{*}$ & 0,182 \\
\hline Subescala de fadiga & $\begin{array}{l}37,14(10,57) \\
(34,91-39,37)\end{array}$ & $\begin{array}{c}44,24(7,96) \\
(42,49-45,98)\end{array}$ & $<0,001^{*}$ & 0,382 \\
\hline
\end{tabular}

Legendas: DP: desvio-padrāo; IC: intervalo de confiança; GCA: grupo câncer; GC: grupo controle; FC: frequência cardíaca; FR: frequência respiratória; PEmax: pressão expiratória máxima; PImax: pressão inspiratória máxima; FPPD: força de preensão palmar direita; FPPE: força de preensão palmar esquerda. Nota: ${ }^{*}<<0,05$, teste de Kruskal-Wallis; $\mathrm{f}$ de Cohen: tamanho de efeito.

Tabela 3. Análise comparativa da subescala de fadiga e das variáveis de força muscular respiratória e periférica de voluntários do grupo câncer que apresentam fraqueza (não aceitável) ou não (aceitável) da musculatura respiratória

\begin{tabular}{|c|c|c|c|c|}
\hline Variáveis & $\begin{array}{l}\text { Aceitável } \\
\text { (n=43) } \\
\text { Média (DP) } \\
\text { (IC 95\%) }\end{array}$ & $\begin{array}{l}\text { Não aceitável } \\
(n=55) \\
\text { Média (DP) } \\
\text { (IC 95\%) }\end{array}$ & Valor de $p$ & d \\
\hline $\operatorname{PEmax}\left(\mathrm{cm} / \mathrm{H}_{2} \mathrm{O}\right)$ & $\begin{array}{c}97,39(18,42) \\
(91,34-103,45)\end{array}$ & $\begin{array}{l}64,47(20,99) \\
(57,93-71,02)\end{array}$ & $<0,001^{*}$ & 1,667 \\
\hline $\operatorname{PImax}\left(\mathrm{cm} / \mathrm{H}_{2} \mathrm{O}\right)$ & $\begin{array}{l}100,68(15,71) \\
(95,52-105,85)\end{array}$ & $\begin{array}{l}48,14(14,48) \\
(43,63-52,65)\end{array}$ & $<0,001^{*}$ & 3,478 \\
\hline FPPD (kgf) & $\begin{array}{l}27,46(11,02) \\
(23,84-31,08)\end{array}$ & $\begin{array}{c}21,74(9,26) \\
(18,85-24,62)\end{array}$ & $0,005^{*}$ & 0,562 \\
\hline FPPE (kgf) & $\begin{array}{l}25,21(10,05) \\
(21,91-28,51)\end{array}$ & $\begin{array}{c}19,36(9,15) \\
(16,50-22,21)\end{array}$ & $<0,001^{*}$ & 0,609 \\
\hline Subescala de fadiga & $\begin{array}{l}36,82(11,84) \\
(32,92-40,71)\end{array}$ & $\begin{array}{l}37,32(10,33) \\
(34,10-40,54)\end{array}$ & 0,960 & 0,045 \\
\hline
\end{tabular}

Legendas: DP: desvio-padrão; IC: intervalo de confiança; PEmax: pressão expiratória máxima; PImax: pressão inspiratória máxima; FPPD: força de preensão palmar direita; FPPE: força de preensão palmar esquerda.

Nota: ${ }^{*}<<0,05$, teste de Mann-Whitney; d de Cohen: tamanho de efeito.

Além disso, esse tratamento é capaz de reduzir as forças musculares periférica e respiratória, sendo identificados poucos estudos sobre o assunto ${ }^{18,42}$.

$\mathrm{Na}$ presença ou não de fraqueza muscular respiratória, a FRC se mantém elevada durante a quimioterapia e/ou a radioterapia. O sintoma de fadiga está relacionado ao próprio câncer e aos efeitos colaterais do seu tratamento, entre os quais, a toxicidade à quimioterapia ${ }^{20} \mathrm{e}$ à radioterapia ${ }^{41}$, o que náo é diferente dos achados do presente estudo. Em 2018, Morishita et al. ${ }^{43}$ também relataram maior fadiga em voluntários com câncer, quando comparados a indivíduos saudáveis, porém não observaram associação com a força muscular periférica.

Os voluntários sem câncer que apresentavam fraqueza muscular respiratória também reduziram a FPP e apresentaram fadiga. A FPP possui associação com a 
Tabela 4. Análise comparativa da subescala de fadiga das variáveis de força muscular respiratória e periférica de voluntários do grupo controle que apresentam fraqueza (não aceitável) ou não (aceitável) da musculatura respiratória

\begin{tabular}{lcccc}
\hline Variáveis & $\begin{array}{c}\text { Aceitável } \\
(\mathbf{n}=68) \\
\text { Média }(\mathbf{D P}) \\
(\mathbf{I C} 95 \%)\end{array}$ & $\begin{array}{c}\text { Não aceitável } \\
(\mathbf{n}=18) \\
\text { Média }(\mathbf{D P}) \\
(\mathbf{I C} 95 \%)\end{array}$ & Valor de $\mathbf{p}$ & $\mathbf{d}$ \\
\hline PEmax $\left(\mathrm{cm} / \mathrm{H}_{2} \mathrm{O}\right)$ & $\begin{array}{c}99,91(22,02) \\
(94,54-105,28)\end{array}$ & $\begin{array}{c}75,65(24,04) \\
(63,28-88,01)\end{array}$ & $<0,001^{*}$ & 1,052 \\
\hline PImax $\left(\mathrm{cm} / \mathrm{H}_{2} \mathrm{O}\right)$ & $\begin{array}{c}102,81(17,82) \\
(98,46-107,15)\end{array}$ & $\begin{array}{c}54,12(10,87) \\
(48,53-59,71)\end{array}$ & $<0,001^{*}$ & 3,299 \\
\hline FPPD $(\mathrm{kgf})$ & $27,57(10,13)$ & $\begin{array}{c}20,00(5,02) \\
(17,41-22,58)\end{array}$ & $0,002^{*}$ & 0,947 \\
\hline FPPE $(\mathrm{kgf})$ & $(25,09-30,04)$ & $\begin{array}{c}19,65(5,89) \\
(16,61-22,68)\end{array}$ & $0,003^{*}$ & 0,825 \\
\hline Subescala de fadiga & $26,13(9,42)$ & $\begin{array}{c}42,17(7,11) \\
(38,52-45,83)\end{array}$ & $0,037^{*}$ & 0,341 \\
\hline
\end{tabular}

Legendas: DP: desvio-padrāo; IC: intervalo de confiança; PEmax: pressão expiratória máxima; PImax: pressão inspiratória máxima; FPPD: força de preensão palmar direita; FPPE: força de preensáo palmar esquerda.

Nota: ${ }^{*} \mathrm{p}<0,05$, teste de Mann-Whitney; d de Cohen: tamanho de efeito.

função pulmonar em adultos jovens e saudáveis ${ }^{44} \mathrm{e}$ em idosos ${ }^{45}$, sendo similar aos achados do presente estudo, em que, quanto maior a força muscular periférica, maior é a força respiratória.

\section{CONCLUSÃO}

O estudo demonstrou que os voluntários com câncer em quimioterapia e/ou em radioterapia apresentam fadiga relatada e redução das forças musculares periférica e respiratória em comparação a voluntários sem câncer. Ao se estratificarem os grupos quanto à força muscular respiratória, identificou-se que voluntários com e sem câncer apresentam o mesmo comportamento respiratório e muscular periférico, porém os indivíduos saudáveis, na presença de fraqueza muscular respiratória, podem denotar fadiga relatada.

O estudo náo teve por objetivo correlacionar as variáveis aos tipos de câncer e aos estádios. Além disso, não foram especificados os tipos de quimioterápicos utilizados no tratamento do câncer e sua relação com as variáveis do estudo. O estudo também não analisou a escala FACT-F, o que pode apresentar algo novo sobre a qualidade de vida de pessoas com câncer e sua relação com as variáveis respiratórias estudadas.

\section{CONTRIBUIÇÕES}

Karina Oliveira Prado Mariano, Ricardo da Silva Alves, Carmélia Bomfim Jacó Rocha, Denise Hollanda Iunes, Juliana Bassalobre Carvalho Borges e Leonardo Cesar Carvalho contribuíram na concepçáo e/ou no planejamento do estudo; na obtenção, análise e interpretação dos dados; assim como na redaçáo e revisão crítica. Ana Paula Aparecida Mantuani e Sabrina Rosse Carvalho contribuíram na concepçáo el ou no planejamento do estudo; e na obtenção, análise e interpretação dos dados. Todos os autores aprovaram a versão final a ser publicada.

\section{DECLARAÇÃO DE CONFLITO DE INTERESSES}

Nada a declarar.

\section{FONTES DE FINANCIAMENTO}

Coordenaçáo de Pessoal de Nível Superior (Capes), Brasil - Código 001 e Programa de Pós-Graduação em Ciências da Reabilitação da Unifal, MG.

\section{REFERÊNCIAS}

1. Bray F, Ferlay J, Soerjomataram I, et al. Global cancer statistics 2018: GLOBOCAN estimates of incidence and mortality worldwide for 36 cancers in 185 countries. CA Cancer J Clin. 2018;68(6):394-424. doi: https:// doi.org/10.3322/caac.21492

2. Arnold M, Sierra MS, Laversanne M, et al. Global patterns and trends in colorectal cancer incidence and mortality. Gut. 2017;66(4):683-91. doi: https://doi. org/10.1136/gutjnl-2015-310912

3. Instituto Nacional de Câncer José Alencar Gomes da Silva. Estimativa 2020: incidência de Câncer no Brasil [Internet]. Rio de Janeiro: INCA; 2019 [acesso 2020 jun 28]. Disponível 
em: https://www.inca.gov.br/sites/ufu.sti.inca.local/ files/media/document/estimativa-2020-incidencia-decancer-no-brasil.pdf

4. Ryan JL, Carroll JK, Ryan EP, et al. Mechanisms of cancer-related fatigue. Oncologist. 2007;12(Suppl 1):2234. doi: https://doi.org/10.1634/theoncologist.12-S1-22

5. Berger AM, Mooney K, Alvarez-Perez A, et al. CancerRelated Fatigue, Version 2.2015. J Natl Compr Canc Netw. 2015;13(8):1012-39. doi: https://doi. org/10.6004/jnccn.2015.0122

6. Humphreys J, de la Maza P, Hirsch S, et al. Muscle strength as a predictor of loss of functional status in hospitalized patients. Nutrition. 2002;18(7-8):616-20. doi: https://doi.org/10.1016/s0899-9007(02)00756-6

7. Novaes RD, Miranda AS, Silva JO, et al. Equaçóes de referência para a predição da força de preensão manual em brasileiros de meia idade e idosos. Fisioter Pesqui. 2009;16(3):217-22. doi: https://doi.org/10.1590/ S1809-29502009000300005

8. Chung CJ, Wu C, Jones M, et al. Reduced handgrip strength as a marker of frailty predicts clinical outcomes in patients with heart failure undergoing ventricular assist device placement. J Card Fail. 2014;20(5):310-5. doi: https://doi.org/10.1016/j.cardfail.2014.02.008

9. Spruit MA, Sillen MJH, Groenen MTJ, et al. New normative values for handgrip strength: results from the UK Biobank. J Am Med Dir Assoc. 2013;14(10):775.e511. doi: https://doi.org/10.1016/j.jamda.2013.06.013

10. Baldwin CE, Bersten AD. Alterations in respiratory and limb muscle strength and size in patients with sepsis who are mechanically ventilated. Phys Ther. 2014;94(1):6882. doi: https://doi.org/10.2522/ptj.20130048

11. Sawada NO, Nicolussi AC, Okino L, et al. Avaliação da qualidade de vida de pacientes com câncer submetidos à quimioterapia. Rev Esc Enferm USP. 2009;43(3):581-7. doi: https://doi.org/10.1590/S008062342009000300012

12. Spyropoulou D, Leotsinidis $M$, Tsiamita $M$, et al. Pulmonary function testing in women with breast cancer treated with radiotherapy and chemotherapy. In Vivo. 2009;23(5):867-71.

13. Savina S, Zaydiner B. Cancer-related fatigue: some clinical aspects. Asia Pac J Oncol Nurs. 2019;6(1):7-9. doi: https://doi.org/10.4103/APJON.APJON_45_18

14. da Silva Alves R, Iunes DH, Pereira IC, et al. Influence of exergaming on the perception of cancer-related fatigue. Games Health J. 2017;6(2):119-26. doi: https://doi. org/10.1089/g4h.2016.0051

15. Oliveira PF, Iunes DH, Alves RS, et al. Effects of exergaming in cancer related fatigue in the quality of life and electromyography of the middle deltoid of people with cancer in treatment: a controlled trial. Asian Pac J Cancer Prev. 2018;19(9):2591-7. doi: https://doi. org/10.22034/APJCP.2018.19.9.2591
16. Abreu APM, Endres D, Costa AB, et al. Função pulmonar e força muscular respiratória em pacientes submetidas à cirurgia oncológica de mama. Rev Bras Cancerol. 2014;60(2):151-7. doi: https://doi.org/10.32635/21769745.RBC.2014v60n2.484

17. Schettino RC, Jotta LMGN, Cassali GD. Função pulmonar em mulheres com câncer de mama submetidas à radioterapia: um estudo piloto. Fisioter Pesqui. 2010;17(3):248-52. doi: https://doi.org/10.1590/ S1809-29502010000300011

18. Celis-Morales CA, Welsh P, Lyall DM, et al. Associations of grip strength with cardiovascular, respiratory, and cancer outcomes and all cause mortality: prospective cohort study of half a million UK Biobank participants. BMJ. 2018;361:K1651. doi: https://doi.org/10.1136/ BMJ.K1651

19. Servaes P, Verhagen C, Bleijenberg G. Fatigue in cancer patients during and after treatment: prevalence, correlates and interventions. Eur J Cancer. 2002;38(1):27-43. doi: https://doi.org/10.1016/s0959-8049(01)00332-x

20. Waseem M, Kaushik P, Tabassum H, et al. Role of mitochondrial mechanism in chemotherapyinduced peripheral neuropathy. Curr Drug Metab. 2018;19(1):47-54. doi: https://doi.org/10.2174/13892 00219666171207121313

21. Suesada MM, Carvalho HA, Albuquerque ALP, et al. Impact of thoracic radiotherapy on respiratory function and exercise capacity in patients with breast cancer. J Bras Pneumol. 2018;44(6):469-76. doi: https://doi. org/10.1590/S1806-37562017000000120

22. American Thoracic Society; European Respiratory Society. ATS/ERS Statement on respiratory muscle testing. Am J Respir Crit Care Med. 2002;166(4):518624. doi: https://doi.org/10.1164/rccm.166.4.518

23. Campbell KL, Winters-Stone KM, Wiskemann $\mathrm{J}$, et al. Exercise guidelines for cancer survivors: consensus statement from international multidisciplinary roundtable. Med Sci Sports Exerc. 2019;51(11):2375-90. doi: https://doi.org/10.1249/MSS.0000000000002116

24. Prinsen H, van Dijk JP, Zwarts MJ, et al. The role of central and peripheral muscle fatigue in postcancer fatigue: a randomized controlled trial. J Pain Symptom Manage. 2015;49(2):173-82. doi: https://doi.org/10.1016/j. jpainsymman.2014.06.020

25. WHO Expert Consultation. Appropriate body-mass index for Asian populations and its implications for policy and intervention strategies. Lancet. 2004;363(9403):157-63. doi: https://doi.org/10.1016/S0140-6736(03)15268-3

26. Porto CC. Semiologia Médica. 8. ed. Rio de Janeiro: Guanabara Koogan; 2019.

27. Koulouris N, Mulvey DA, Laroche CM, et al. Comparison of two different mouthpieces for the measurement of Pimax and Pemax in normal and weak subjects. Eur Respir J. 1988;1(9):863-7. 
28. Azeredo CAC. Fisioterapia respiratória moderna. São Paulo: Manole; 2002.

29. Ashton L, Myers S. Serial grip strength testing- its role in assessment of wrist and hand disability. Internet J Surg. 2003;5(2).

30. Ishikawa NM, Thuler LCS, Giglio AG, et al. Validation of the portuguese version of Functional Assessment of Cancer Therapy-Fatigue (FACT-F) in Brazilian cancer patients. Support Care Cancer. 2010;18(4):481-90. doi: https://doi.org/10.1007/s00520-009-0697-0

31. Cella D, Lai JS, Chang CH, et al. Fatigue in cancer patients compared with fatigue in the general United States population. Cancer. 2002;94(2):528-38. doi: https://doi.org/10.1002/cncr.10245

32. Cohen J. Statistical power analysis for the behavioral sciences. 2nd ed. New Jersey: Lawrence Erlbaum; 1988. doi: https://doi.org/10.4324/9780203771587

33. Lindenau JDR, Gimarães LSP. Calculando o tamanho de efeito no SPSS. Rev HCPA. 2012;32(3):363-81.

34. Dolci A, Dominici R, Cardinale D, et al. Biochemical markers for prediction of chemotherapy-induced cardiotoxicity: systematic review of the literature and recommendations for use. Am J Clin Pathol. 2008;130(5):688-95. doi: https://doi.org/10.1309/ AJCPB66LRIIVMQDR

35. Suter TM, Ewer MS. Cancer drugs and the heart: Importance and management. Eur Heart J. 2013;34(15):1102-11. doi: https://doi.org/10.1093/ eurheartj/ehs 181

36. Coelho CM, Carvalho RM, Gouvêa DSA, et al. Comparação entre parâmetros de pressóes respiratórias máximas em indivíduos saudáveis. J Bras Pneumol. 2012;38(5):605-13. doi: https://doi.org/10.1590/ S1806-37132012000500010

37. Santos DE, Rett MT, Mendonça ACR, et al. Efeito da radioterapia na função pulmonar e na fadiga de mulheres em tratamento para o câncer de mama. Fisioter Pesqui. 2013;20(1):50-5. doi: https://doi.org/10.1590/S180929502013000100009

38. Reidunsdatter RJ, Rannestad T, Frengen J, et al. Early effects of contemporary breast radiation on health-related quality of life - Predictors of radiotherapy-related fatigue. Acta Oncol. 2011;50(8):1175-82. doi: https://doi.org/1 0.3109/0284186X.2011.604345

39. Ruiz JR, Sui X, Lobelo F, et al. Association between muscular strength and mortality in men: prospective cohort study. BMJ. 2008;337(7661):a439. doi: https:// doi.org/10.1136/bmj.a439

40. Caporrino FA, Faloppa F, Santos JBG, et al. Estudo populacional da força de preensão palmar com dinamômetro Jamar ${ }^{\circledR}$. Rev Bras Ortop. 1998;33(2):1-1.

41. Ebede CC, Jang Y, Escalante CP. Cancer-related fatigue in cancer survivorship. Med Clin North Am.
2017;101(6):1085-97. doi: https://doi.org/10.1016/j. mcna.2017.06.007

42. Steemburgo T, Averbuch NC, Belin CHS, et al. Hand grip strength and nutritional status in hospitalized oncological patients. Rev Nutr. 2018;31(5):489-99. doi: https://doi.org/10.1590/1678-98652018000500006

43. Morishita S, Tsubaki A, Fu JB, et al. Cancer survivors exhibit a different relationship between muscle strength and health-related quality of life/fatigue compared to healthy subjects. Eur J Cancer Care (Engl). 2018;27(4):e12856. doi: https://doi.org/10.1111/ ECC. 12856

44. Mgbemena NC, Aweto HA, Tella BA, et al. Prediction of lung function using handgrip strength in healthy young adults. Physiol Rep. 2019;7(1):e13960. doi: https://doi. org/10.14814/phy2.13960

45. Bahat G, Tufan A, Ozkaya H, et al. Relation between hand grip strength, respiratory muscle strength and spirometric measures in male nursing home residents. Aging Male. 2014;17(3):136-40. doi: https://doi.org/1 $0.3109 / 13685538.2014 .936001$ 